\title{
Predicting engagement with services for psychosis: insight, symptoms and recovery style
}

\author{
LYNDA TAIT, MAX BIRCHWOOD and PETER TROWER
}

\begin{abstract}
Background Treatment nonadherence and service disengagement are commonly attributed to impaired insight. There is evidence that recovery style (i.e. psychological adjustment) may underlie service engagement.
\end{abstract}

Aims We examined whether insight, psychotic symptoms or individuals' recovery style ('integration' v. 'sealingover') predicts service engagement.

\section{Method Fifty patients with schizophrenia were assessed during acute psychosis and at 3-month and 6-month follow-ups. Measures included recovery style, psychosis symptoms, insight and service engagement.}

Results Sealing-over at 3 months following onset of an episode of psychosis predicted low service engagement at 6 months. Neither insight nor symptoms predicted engagement. The clear shift from integration to sealing-over within the first 3 months was independent of changes in symptoms or insight. Sealing-over between 3 and 6 months was associated with improvement in psychosis symptoms.

Conclusions Recoverystyle contributed more to engagement than did insight, appears to be dynamic in the short term and is orthogonal to insight. Overall, this study demonstrated the importance of addressing psychological adjustment to psychosis as well as illness status when investigating treatment engagement in people with psychosis.

Declaration of interest None.
Individuals with psychosis are often difficult to engage in mental health services (e.g. Sainsbury Centre for Mental Health, 1998), with consistent evidence that up to $80 \%$ are non-adherent to treatment (Corrigan et al, 1990). Failure to engage with services and to adhere to treatment is commonly attributed to lack of insight (e.g. Ghaemi \& Pope, 1994); however, evidence for a relationship between insight and treatment adherence is inconclusive (e.g. Trauer \& Sacks, 2000). One explanation is that psychological adjustment to psychosis might underlie these difficulties (McGlashan \& Levy, 1977). However, the study of psychological adjustment to psychosis has been limited by the focus on impaired insight and acceptance of treatment. Further understanding of nonengagement with services or treatment might assist in the development of interventions to enhance engagement and treatment adherence (e.g. Kemp et al, 1996). This is an important issue because of the risk of deterioration without longterm treatment and the recent emphasis on targeting difficult-to-engage clients (Department of Health, 2000).

\section{Background}

Impaired insight might reflect, in part, a psychological process of denial as a way of coping with the negative consequences of illness (Johnson \& Orrell, 1996). Denial of the need for services and refusal to participate in treatment may represent an avoidance strategy as a means of dealing with the stigmatising consequences of psychosis. One such coping strategy or recovery style is 'sealing-over' (McGlashan et al, 1977), which is a way of coping by minimising the significance of symptoms and the impact of psychosis and showing a lack of curiosity about the experience. However, sealing-over has been viewed as either an epiphenomenon of lack of insight or as synonymous with it (e.g. Amador et al,
1991; Dixon et al, 1998). One study that has addressed this issue found no relationship between insight and recovery style (Drayton et al, 1998); however, it is possible that recovery style and insight do have a clinically meaningful relationship. Furthermore, the conceptualisation of integration and sealing-over suggests that they represent enduring personality trait characteristics (McGlashan, 1987), which implies that they are more or less stable over time. This is a challenge for therapeutic interventions that aim to help individuals to progress from sealing-over to integration (e.g. Jackson et al, 1998). In this study, we aim to determine to what extent engagement with services arises from impaired insight, symptoms of psychosis or recovery style; and, in relation to recovery style, whether this is a stable trait and related to insight.

\section{Hypotheses}

(a) Individuals who report sealing-over recovery styles will reveal low engagement with services, which will be independent of insight or symptoms.

(b) There will be significant changes in recovery style over time, representing the process of psychological adjustment to psychosis, and recovery style will be independent of insight or symptoms.

\section{METHOD}

\section{Sample and procedure}

All patients who were receiving treatment for acute psychosis as in-patients or during home treatment were considered for recruitment from two urban mental health services. Patients had an ICD-10 (World Health Organization, 1992) chart diagnosis of schizophrenia or related disorders (F20, F22, F23, F25) and a history of either multiple episodes or a single episode of psychosis. Patients were interviewed at intake using the Structured Clinical Interview for the Positive and Negative Syndrome Scale (SCI-PANSS; Kay et al, 1987) to confirm the chart diagnosis. Patients with a primary diagnosis of substance use disorder, mood disorder or organic mental disorder were excluded. Patients meeting inclusion criteria and providing written consent were interviewed as soon as possible after admission to hospital or the start of home treatment for acute psychosis, with all patients assessed within 3 weeks of the start of treatment. All patients were 
reinterviewed at 3 and 6 months following the initial interview. Patients were informed that the purpose of the study was to assess the different ways in which people cope during recovery from an episode of psychosis, but they were unaware of the study's specific hypotheses.

\section{Design}

This was a prospective longitudinal study in which three assessments were undertaken: during an acute episode of psychosis and at 3 and 6 months following the initial interview.

\section{Assessments}

\section{Recovery style}

Recovery style was measured with the Recovery Style Questionnaire (RSQ Drayton et al, 1998). The RSQ is a 39-item self-report measure specifically designed as an alternative to the lengthy interview measure of recovery style pioneered by McGlashan et al (1977). As described by McGlashan et al, the concept of an 'integration' recovery style is characterised by acknowledgement of, and curiosity about, the significance of psychosis and by active attempts to cope in managing the illness. By contrast, a 'sealing-over' recovery style is characterised by cognitive and behavioural avoidance of the diagnosis and experience of psychosis.

The RSQ consists of 13 sub-scales, each containing three questions designed to reflect categories consistent with those developed by McGlashan et al. The 13 sub-scales of the RSQ are: (1) Curiosity, to investigate if the person has 'curiosity about psychotic experiences and is invested in understanding them'; (2) Education, to investigate if 'the individual treats the psychosis as a source of new information about himself/herself'; (3) Optimism, to identify if 'the individual is able to see positive aspects to having had a psychotic episode'; (4) Impact, to evaluate the person's reaction to the 'psychotic experience having had a strong impact on life'; (5) Fear, to identify 'the individual's attitude toward mental illness'; (6) Liking, to identify if 'the individual liked some of the unusual feelings and ideas experienced during psychosis'; (7) Continuity, to analyse the 'awareness of continuity between thoughts and feelings during the psychosis and emotional conflicts prior and subsequent to the psychotic episode'; (8) Ownership, to investigate if the 'psychosis is experienced by the individual as personal, rather than as alien'; (9) Responsibility, to identify if 'the individual feels responsible for psychotic thoughts and acts'; (10) Helpseeking, to evaluate if 'the individual enlists the help of others in mastering the conflicts related to psychosis'; (11) Blame, to investigate if 'the individual blames the illness on own difficulties in coping with life'; (12) Cause, to evaluate if 'the individual views himself/herself as having had an emotional or nervous breakdown'; (13) Satisfaction, to evaluate the person's views that 'psychosis helped in obtaining satisfaction in life'.

Scoring of the RSQ allows the classification of four recovery styles: (1) integration; (2) mixed picture in which integration predominates; (3) mixed picture in which sealing-over predominates; and (4) sealing-over. Higher scores represent sealing-over. The RSQ has been shown to have excellent psychometric properties and was validated against the interview version (Drayton et al, 1998).

\section{Service engagement}

Service engagement was measured with the Service Engagement Scale (Tait et al, 2002). No special training is required to use this brief measure. The Service Engagement Scale is a 14-item measure consisting of statements that assess client engagement with services, which case managers rate on a four-point Likert scale from 'not at all or rarely' to 'most of the time'. The total score ranges from a minimum of zero to a maximum of 42. Higher scores indicate lower engagement. Four sub-scales assess availability ('When a visit is arranged, the client is available'), collaboration ('The client actively participates in managing his/her illness'), help-seeking ('The client seeks help to prevent a crisis') and treatment adherence ('The client refuses to cooperate with treatment'). The scale has high internal consistency and retest reliability, including discrimination between criterion groups, in an assertive outreach team (Tait et al, 2002).

\section{Insight}

Insight was measured with the Insight Scale (Birchwood et al, 1994). This 8-item selfreport scale was designed to be sensitive to changes in levels of insight, and captures each of three widely accepted dimensions of insight: perceived need for treatment, awareness of illness and relabelling of symptoms as pathological. Higher scores indicate greater levels of insight. The psychometric properties of the scale are excellent and it is widely used in psychosis research.

\section{Psychotic symptoms}

Psychotic symptoms were measured with the SCI-PANSS (Kay et al, 1987). The 30item SCI-PANSS is a widely used, valid and reliable measure for assessing mental state.

The RSQ, Insight Scale and SCI-PANSS were administered at each of the three assessments. Case managers completed the Service Engagement Scale at the 6-month assessment.

\section{Data analysis}

One-way ANOVAs, with planned comparisons, were used to assess recovery style group differences in mean sub-scale and total Service Engagement Scale scores and mean total insight and PANSS scores at each time point. Pearson correlations were used to examine associations between engagement scores, insight and symptoms of psychosis, and Spearman correlations were used to examine associations between recovery style, insight and PANSS change scores. For the second hypothesis, repeated-measures ANOVAs were used to determine the stability of recovery style longitudinally, using the multivariate analysis of variance (MANOVA) approach (Tabachnick \& Fidell, 2001). Repeatedmeasures one-way MANOVAs with linear and quadratic trends over time were performed to examine the patterns of change in the levels of insight and symptoms of psychosis longitudinally.

\section{RESULTS}

\section{Sample characteristics}

Sixty-two individuals were considered for entry to the study, 12 of whom declined to participate: a response rate of $81 \%$. Reasons for refusal were not sought nor demographic data obtained in line with ethical approval. Attrition rates were low, with eight $(16 \%)$ participants lost to the study. Fifty participants who met the ICD-10 diagnostic criteria for schizophrenia were interviewed, and complete follow-up data were obtained for 46 participants at the second assessment and for 42 participants at the third assessment. 
No significant differences were found for comparison of those who completed all three assessments with those who were lost to the study on gender, ethnic group, age, living situation or recovery style scores. There were no significant differences in any of the clinically relevant baseline measures between participants and those lost to follow-up (duration of illness, PANSS total scores, insight).

The mean age of the participants was 33.8 years (s.d. $=12.0)$; the group comprised $19(38 \%)$ females and 31 (62\%) males. They were predominantly single $(70 \%)$ and living alone $(58 \%)$. The mean duration of illness was 7.2 years (s.d.=9.71), with a wide range of 2 days to 36 years, and $68 \%$ of the sample had been ill for 5 years or less.

\section{Engagement, recovery style, insight and symptoms}

The minimum possible Service Engagement Scale score of zero was scored by three participants $(8.6 \%) ; 60 \%$ scored above the criterion for 'low' engagement (11 points or above; Tait et al, 2002).

To test the first hypothesis - that the sealing-over recovery style would predict lower engagement than the integration recovery style - a set of one-way betweengroup ANOVAs with pre-planned contrasts was performed on the Service Engagement Scale. Recovery style scores at the 3-month follow-up were used to predict engagement assessed at the 6-month follow-up. For this analysis, planned comparisons involved comparing the means of the two integration groups combined ('integration' and 'mixedintegration') with that of the two sealingover groups combined ('sealing-over' and 'mixed sealing-over'). These data are presented in Table 1. It should be noted that high scores are indicative of low engagement. Contrasting individuals with integration recovery styles $v$. individuals with sealing-over recovery styles on each of the four sub-scales and total scale scores of the Service Engagement Scale indicated significantly lower engagement in the sealing-over recovery style groups relative to the integration recovery style groups (see Table 1).

There were significant between-group differences for total Service Engagement Scale ratings $(F(3,31)=8.04, \quad P<0.001$, $\left.\eta^{2}=0.44\right)$. Thus, recovery style at 3 months after acute psychosis predicted the level of engagement with services at 6 months. Among those with sealing-over recovery styles, $6.3 \%$ were rated as 'high' engagement compared with $52.6 \%$ of those with integration recovery styles.

Insight measured at 3 months showed no significant correlation with the Service Engagement Scale scores measured at 6 months $(r=-0.09$, NS). Similarly, the PANSS negative $(r=0.16$, NS), PANSS positive $(r=0.23, \mathrm{NS})$ and PANSS general psychopathology $(r=0.07, \quad \mathrm{NS})$ scores measured at 3 months showed no significant correlations with the Service Engagement Scale scores measured at 6 months. Thus, Insight Scale and PANSS scores at 3 months did not predict the Service Engagement Scale scores at 6 months. There was also no correlation between Service Engagement Scale scores and Insight Scale and PANSS scores at 6 months or changes in Insight Scale and PANSS scores during the follow-up.

\section{Are changes in insight and symptoms of psychosis linked to recovery style?}

In order to understand further the nature of recovery style, changes in insight and symptoms of psychosis over time were examined. According to Birchwood et al (1994), an insight score of 9-12 represents 'high insight'. During the acute episode, $48 \%$ of participants scored 9-12 on the Insight Scale and the majority of participants at 3 months $(63 \%)$ were in the 9-12 range of scores, indicating that level of insight at each of the data points was high for many individuals.

Mean insight scores at baseline and at 3- and 6-month follow-ups were 7.8 $($ s.d. $=3.2), \quad 9.2 \quad($ s.d. $=2.0) \quad$ and $\quad 8.8$ (s.d.=2.0). Mean total insight scores were compared across three assessments by one-way repeated-measures MANOVA. The results revealed a significant main effect for time (Wilks' $\lambda=0.84, F(2,40)$ $\left.=3.95, P=0.03, \eta^{2}=0.17\right)$. A quadratic trend was significant $(F(1,41)=7.78$, $\left.P=0.008, \eta^{2}=0.16\right)$, indicating an increase in insight scores between baseline and 3 months but then showing a slight mean decrease between 3 and 6 months.

Mean PANSS scores at baseline and at 3- and 6-month follow-ups were 54.2 (s.d.=11.5), $38.8 \quad$ (s.d.=7.5) and 34.5 (s.d.=4.6). Mean total PANSS scores were compared across three assessments by one-way repeated-measures MANOVA, revealing a significant main effect for time (Wilks' $\lambda=0.24, F(2,40)=62.38, P=0.001$, $\left.\eta^{2}=0.76\right)$. There were also significant linear $\left(F(1,41)=124.74, P=0.001, \eta^{2}=0.75\right)$ and quadratic $\quad(F(1,41)=22.08, \quad P=0.001$, $\left.\eta^{2}=0.35\right)$ trends, indicating a sharp drop in symptoms of psychosis between baseline and 3 months, followed by a more moderate decrease over time between 3 and 6 months, in line with expectations concerning clinical recovery.

One-way ANOVAs were conducted on the total scores for the PANSS and the Insight Scale in order to test for differences between recovery style groups at each time point. No significant differences emerged between the four recovery style groups for either of the two variables at any time point, with means for each of the recovery style groups being very similar.

Table I Means (standard deviations) of scores on the Service Engagement Scale sub-scales and total scale scores for recovery style groups

\begin{tabular}{|c|c|c|c|c|c|c|}
\hline \multirow{2}{*}{$\begin{array}{l}\text { Service Engagement Scale } \\
\text { sub-scales at } 6 \text { months }\end{array}$} & \multicolumn{4}{|c|}{ Recovery style groups at 3 months } & \multicolumn{2}{|c|}{ Contrast } \\
\hline & Integration & Mixed-integration & Mixed-sealing-over & Sealing-over & $F$ & $P$ \\
\hline Availability & $1.5(1.9)$ & $1.7(2.2)$ & $4.3(2.9)$ & $4.6(3.2)$ & 10.61 & 0.002 \\
\hline Collaboration & $2.5(2.5)$ & $3.7(3.2)$ & $7.3(2.2)$ & $6.7(1.3)$ & 21.73 & 0.001 \\
\hline Help-seeking & $2.0(2.9)$ & $5.1(3.4)$ & $8.6(4.2)$ & $9.6(2.4)$ & 25.21 & 0.001 \\
\hline Treatment adherence & $1.8(2.6)$ & $2.9(3.2)$ & $5.7(2.7)$ & $5.9(3.1)$ & 12.12 & 0.001 \\
\hline Total scale score & $7.8(8.8)$ & $13.3(10.8)$ & $25.9(I I . I)$ & $26.7(8.9)$ & 21.83 & 0.001 \\
\hline
\end{tabular}


No significant correlations emerged when changes in recovery style were plotted against changes in insight at 3 and 6 months, or in PANSS change scores at 3 months. However, the change in psychotic symptoms between 3 and 6 months was correlated $(r=-0.34, P=0.03)$ with the RSQ change scores at 6 months, indicating that improvement in psychotic symptoms was associated with an increased tendency towards sealing-over. These results suggest that insight is independent of recovery style. Thus, no evidence was found to support the notion that sealing-over is synonymous with lack of insight.

\section{Stability of recovery style over time}

The most widely used coping styles at baseline were the two integration recovery styles $(72 \%)$ but by the 6 -month followup the most widely used were the two sealing-over recovery styles $(59.5 \%)$. To test the second hypothesis, mean recovery style scores were compared across time by one-way repeated-measures MANOVA. The mean (s.d.) recovery style scores longitudinally were: baseline $=2.98$ (s.d. $=1.22$ ), 3 months $=3.52$ (s.d.=1.38) and 6 months $=3.69$ (s.d.=1.51). The results of the analysis support the prediction by showing a large significant main effect of time (Wilks' $\lambda=0.80, F(2,40)=5.07, P=0.011$, $\left.\eta^{2}=0.20\right)$. The results of the polynomial trend analysis support the prediction by showing that the linear trend was significant $\left(F(1,41)=10.39, P=0.002, \eta^{2}=0.20\right)$, indicating that recovery style scores increased across time towards sealing-over. However, very little change in recovery style occurred between 3 and 6 months.

\section{DISCUSSION}

To date, there has been no investigation of the relationship between engagement with mental health services and individuals' psychological adjustment to the diagnosis $v$. insight and symptoms of psychosis.

\section{Engagement, recovery style, insight and symptoms}

The sealing-over recovery styles were associated with considerably lower service engagement than the integration recovery styles, with $46 \%$ more engagement in the integration groups. Furthermore, recovery style at 3 months predicted service engagement at 6 months, and there was no relationship between service engagement and either psychotic symptoms or insight. To recap, those who endorsed sealing-over recovery styles were rated by their case managers as more likely to have difficulty in arranging and keeping appointments, in developing a collaborative relationship with mental health services staff, in seeking help in a crisis and in adhering to prescribed treatments. These results support the proposition that coping and adjustment to psychosis are among the main influences on how service users interact with statutory services. Those individuals who have avoidance-type coping behaviours (i.e. sealing-over) in the context of psychosis are more likely also to avoid or withdraw from care provided by mental health professionals, whether insight is high or otherwise. Understanding the relationship between recovery style and engagement will be a step forward in designing interventions to enhance engagement with services.

\section{Recovery style and insight}

There was no evidence to support the notion that sealing-over is synonymous with lack of insight, consistent with the finding of Drayton et al (1998). It is possible, therefore, for patients to seal-over with or without insight, e.g. 'I know I am ill but it was just one of those things and I want to forget about it and move on'. One client, for example, had high insight at 6 months (Insight Scale score $=12$ ) but used a sealing-over recovery style (recovery style score $=6$ ) and reported that the illness had little effect on his life. This client was rated by the case manager as rarely seeking help in a crisis or taking an active role in managing his illness, as sometimes avoiding contact and as someone who maintained a low level of adherence to treatment throughout the study period.

\section{Adjustment to psychosis}

The results support the second hypothesis in suggesting that, for a subgroup, recovery style has a distinct capacity for change, supporting earlier research that for some people with psychosis the recovery style appears to measure the psychological adjustment to the illness (Jackson et al, 1998) and is inconsistent with earlier suggestions that recovery style may be solely a trait characteristic (McGlashan, 1987). Recovery style showed clear evidence of moving towards sealing-over during the early phase of adjustment to an episode of psychosis. It is important to emphasise that the temporal trend for recovery style was in the opposite direction to that observed for insight and symptoms (insight and symptoms improved with time). It is possible that when insight and symptoms improve, individuals become more aware of the impact and negative consequences of psychosis, motivating sealing-over. The finding that many individuals adopt 'integrating' recovery styles initially during active treatment may reflect attempts to cope constructively with the challenge of the situation. However, during the course of clinical recovery, the opportunity for reflection begins, which may explain the clear shift towards sealing-over.

In previous research (Birchwood et al, $2000 a, b)$ we have demonstrated that individuals' appraisals of psychosis and the implications for their future and identity are critical for recovery and that they predict later depression, particularly the appraisal of loss ('I will never be the person I was') or shame ('If people find out, I will be humiliated'). It is not yet understood why some clients use sealing instead of integrating strategies, although we believe that patients who seal are those who see greater loss and shame in their psychosis, motivating denial (Birchwood et al, 2000b). In previous work, 'sealers' were found to have vulnerable self-esteem and a history of insecure attachment, seemingly unable to face up to the personal impact of a psychosis (Drayton et al, 1998). We are currently exploring this possibility further and how it might interact with parallel processes in relatives.

\section{Methodological issues}

The participants in the present study were located in mental health facilities within urban and inner-city settings. Although there is no a priori reason to suppose that such settings are different from rural settings, ethnicity and social disadvantage could, conceivably, influence the process of adaptation to psychosis. It is important also to bear in mind that this sample was, in $7 / 10$ cases, within 5 years of onset of illness and the experimental sampling method identified patients in acute crisis, which will engage a significant group of multiple relapsers. Although this is representative of the trajectories of psychosis in general (Harrison et al, 2001), undoubtedly there will be interactions between the course of 
psychosis and individual processes of adaptation; the process of adaptation begins at the first episode or even earlier. In a nutshell, what we have observed is a 6-month snapshot of a group of patients who are prone to relapse where sealing has become an endemic part of their attitude to their illness, leading to service disengagement and treatment reluctance.

The researcher who conducted the PANSS assessments was not blind to two of the assessments - insight and recovery style - and this may be a source of potential bias; however, both insight and recovery style were assessed by self-report measures, which were not scored until the conclusion of the study, and this limited the potential for bias.

In general, the rate of attrition in this study was low $(16 \%)$ and there were no notable differences in demographic, recovery style or clinical data between those who dropped out and those who completed all three assessments. This suggests that little specific bias was introduced by those who dropped out posing a threat to the internal validity of the study. However, it is acknowledged that the 12 individuals who refused to participate might have used sealing-over recovery styles and thus introduced a degree of bias into the final sample studied. We would emphasise, however, that the study included a high percentage of poor engagers and individuals who used sealing-over recovery styles. Thus, we believe that the overall conclusions are valid and that the internal validity of the study was not threatened.

\section{Clinical implications}

First and foremost, these data suggest that sealing/integration and not insight/ symptoms lie at the heart of service disengagement and treatment reluctance. 'Compliance therapy' (Kemp et al, 1996) a motivational approach to improving drug adherence - may have achieved its results not through modifying insight but by intervening at the level of the individual's psychological defences to treatment, which we believe to be synonymous with the concept of sealing. Notable also in the Kemp et al study was that the sample characteristics were similar to ours and samples were recruited at the acute episode.

Second, the naturalistic changes that we have observed in recovery style suggest that providing appropriate support at an early stage may help to maintain an integrating

\section{CLINICAL IMPLICATIONS}

Following symptomatic recovery, 'sealing-over' begins, suggesting the importance of early support.

- Assessment of recovery style may benefit attempts to predict and enhance engagement with services.

- This study suggests that interventions designed to promote psychological adjustment may not have to wait for full remission of psychosis symptoms or for full insight to be achieved.

\section{LIMITATIONS}

- The results may be limited to urban and inner-city settings.

- The interview and self-report assessments were not blind, but were independent.

- Although the refusal rate was low, we do not know if those who declined to participate used a sealing-over recovery style and thus introduced an element of volunteer bias.

LYNDA TAIT, PhD, School of Psychology, University of Birmingham; MAX BIRCHWOOD, PhD, FBPsS, Early Intervention Service, Northern Birmingham Mental Health NHS Trust and School of Psychology, University of Birmingham; PETER TROWER, PhD, School of Psychology, University of Birmingham, UK

Correspondence: Lynda Tait, School of Psychology, University of Birmingham, Edgbaston, Birmingham BI5 2TT,UK. E-mail: lat726@bham.ac.uk

(First received 2 May 2002, final revision 3 October 2002, accepted 10 October 2002)

response in recovering from psychosis, and that initial sealing-over might be amenable to change. The evidence from this study and cognitive interventions (Jackson et al, 1998) provides support for this possibility. However, it is likely that multiple factors influence the subjective impact of psychosis and the person's psychological adjustment to it (Jackson et al, 1999). In addition to cognitive appraisal (Birchwood et $a l, 2000 a, b)$, it is likely that the family response (Patterson et al, 2000) and psychological vulnerabilities (Drayton et al, 1998) influence how well individuals will adjust to psychosis over time.

We are currently examining the relationship between recovery style and several interpersonal and psychological vulnerability variables that may underpin or maintain recovery style. The findings of the current study provide support for the development of interventions such as compliance therapy and cognitive therapy, designed to promote psychological adjustment to psychosis. Efforts to enhance engagement with mental health services may also benefit from an assessment of a person's recovery style. Clinicians may be able to tailor their interventions with patients according to their current recovery style. In this way, engagement, effective coping strategies and successful psychological adjustment may be promoted. Finally, the finding that recovery style is independent of insight suggests that interventions to promote psychological adjustment to psychosis need not wait for full insight to be achieved or for complete remission of symptoms.

\section{ACKNOWLEDGEMENTS}

We thank the participants and support staff in the study for their cooperation and commitment to completion of the study.

\section{REFERENCES}

Amador, X. F., Strauss, D. H., Yale, S. A., et al (1991) Awareness of illness in schizophrenia. Schizophrenic Bulletin, 17, 113-132. 
Birchwood, M., Smith, J., Drury, V., et al (1994) A self-report insight scale for psychosis: reliability, validity and sensitivity to change. Acta Psychiatrica Scandinavica, 89, 62-67.

—, Iqbal, Z., Chadwick, P., et al (2000a) Cognitive approach to depression and suicidal thinking in psychosis. I: Ontogeny of post-psychotic depression. British Journal of Psychiatry, 177, 516-521.

$\ldots, \ldots, \ldots$, et al (2000b) Cognitive approach to depression and suicidal thinking in psychosis. 2: Testing the validity of a social ranking model. British Journal of Psychiatry, 177, 522-528.

Corrigan, P. W., Liberman, R. P. \& Engel, J. D. (1990) From non-compliance to collaboration in the treatment of schizophrenia. Hospital and Community Psychiatry, 4I, 1203-1211.

Department of Health (2000) The NHS Plan: a Plan for Investment, a Plan for Reform. London: Department of Health.

Dixon, M., King, S. \& Steiger, H. (1998) The contribution of depression and denial towards understanding the unawareness of symptoms in schizophrenic out-patients. British Journal of Medical Psychology, 7I, 85-97.

Drayton, M., Birchwood, M. \& Trower, P. (1998) Early attachment experience and recovery from psychosis. British Journal of Clinical Psychology, 37, 269-284.
Ghaemi, S. N. \& Pope, H. G. (1994) Lack of insight in psychotic and affective disorders: a review of empirical studies. Harvard Review of Psychiatry, 2, 22-33.

Harrison, G., Hopper, K., Craig, T., et al (200I) Recovery from psychotic illness: a 15- and 25-year international follow-up study. British Journal of Psychiatry, 178, 506-517.

Jackson, H., McGorry, P., Edwards, J., et al (1998) Cognitively-oriented psychotherapy for early psychosis (COPE). Preliminary results. British Journal of Psychiatry, 172 (suppl. 33), 93-100.

Jackson, H. J., Edwards, J., Hulbert, C., et al (1999) Recovery from psychosis: psychological interventions. In The Recognition and Management of Early Psychosis: a Preventive Approach (eds P. D. McGorry \& H. J. Jackson), pp. 265-307. Cambridge: Cambridge University Press.

Johnson, S. \& Orrell, M. (1996) Insight, psychosis and ethnicity: a case-note study. Psychological Medicine, $\mathbf{2 6}$ 1081-1084.

Kay, S. R., Fiszbein, A. \& Opler, L. A. (1987) The positive and negative syndrome scale (PANSS) for schizophrenia. Schizophrenia Bulletin, 13, 26I-276.

Kemp, R., Hayward, P., Applewhaite, G., et al (1996) Compliance therapy in psychotic patients: randomised controlled trial. BMJ, 312, 345-349.

McGlashan, T. H. (1987) Recovery style from mental illness and long-term outcome. Journal of Nervous and Mental Disease, 175, 68I-685.
— \& Levy, S. T. (1977) Sealing-over in a therapeutic community. Psychiatry, 40, 55-65.

_ , Wadeson, H. S., Carpenter, W. T., et al (1977) Art and recovery style from psychosis. Journal of Nervous and Mental Disease, 164, 182-190.

Patterson, P., Birchwood, M. \& Cochrane, R. (2000) Preventing the entrenchment of high expressed emotion in first episode psychosis: early developmental attachment pathways. Australian and New Zealand Journal of Psychiatry, 34 (suppl.), SI9I-SI97.

Sainsbury Centre for Mental Health (1998) Keys to Engagement: Review of Care for People with Severe Mental Illness who are Hard to Engage with Services. London: Sainsbury Centre Publications.

Tabachnick, B. G. \& Fidell, L. S. (200I) Using Multivariate Statistics. Boston, MA: Allyn \& Bacon.

Tait, L., Birchwood, M. \& Trower, P. (2002) A new scale (SES) to measure engagement with community mental health services. Journal of Mental Health, II, 191-198.

Trauer, T. \& Sacks, T. (2000) The relationship between insight and medication adherence in severely mentally ill clients treated in the community. Acta Psychiatrica Scandinavica, 102, 211-216

World Health Organization (1992) The ICD-10 International Classification of Diseases and Related Health Problems (Tenth Revision). Geneva: WHO. 